\title{
When an Incompressible Ocean Impacts a Compressible Continent
}

\author{
Kern E. Kenyon \\ 4632 North Lane, Del Mar, USA \\ Correspondence to: Kern E. Kenyon, kernken@aol.com \\ Keywords: Tidal Reflection, Continental Compression \\ Received: June 23, $2021 \quad$ Accepted: July 12, 2021
}

Published: July 15, 2021

Copyright () 2021 by author(s) and Scientific Research Publishing Inc.

This work is licensed under the Creative Commons Attribution International License (CC BY 4.0).

http://creativecommons.org/licenses/by/4.0/

\section{(c) (i) Open Access}

\section{ABSTRACT}

The horizontal force on a continent, at the land/ocean boundary, is calculated according to the concept of reflected tide waves, in which there is a time rate of change (reversal) of the linear momentum brought in by the tide to the continent. From the text books, the Stokes drift for surface gravity waves propagating in arbitrary constant mean depth, which is directly related to the linear momentum of the wave, is adapted to the tide wave by using the approximation that the wavelength of the tide is very much larger than the vertical length of the water column. This horizontal force is a maximum at full and new moon when the tide amplitude is greatest. Evidence is cited for the correlation of earthquakes at full moons. The tendency of the reflected tide force on the continents is to cause compression of the solid material in the horizontal direction and it is suggested that this possibly could trigger earthquakes occasionally.

\section{INTRODUCTION}

Evidence that continents can be considered compressible has been tied for three hundred years to the knowledge that the earth's equatorial radius is significantly larger than the polar radius, and the thought that the root cause of this fact is due to the daily rotation of the earth about its axis plus the outward centrifugal force.

Among the various ways an ocean can be put into motion relative to the solid earth, the most commonly found are by: the wind generated gyre circulations, thermohaline processes and the tides. When an encounter occurs between moving ocean water and a continent standing still, if any compression is expected to take place, it must be in the solid because the fluid is incompressible.

When my daughter was away in college in Santa Cruz, CA, a major earthquake took place there. Somehow I became aware that there was a full moon at the time. The year was 1989. The quake was on October 17 and the moon was full on October 14! Ever since then I have checked every large or major earthquake in the world for such a coincidence, within a day or so, and enough correlations have built up 
over time to be pleasing, in the sense that more attention to the problem appeared to be needed, although unfortunately the different cases were not recorded by me. Apparently, there can be such a connection between the tides and the continents.

\section{TIDES}

Ocean tides are generated by the gravitational attractions of the sun and moon and they propagate like shallow water surface gravity waves even in the deepest parts of the oceans. Like all surface gravity waves they carry linear momentum with them, which is directly related to the Stokes drift [1]: a small net movement of the fluid particles in the wave propagation direction (the orbital particle movement occurs in circles or ellipses that are not quite closed).

Upon reflection from a vertical wall a surface gravity wave imparts a force to the wall that is straightforwardly related to the time rate of change (reversal) of the linear momentum carried by the wave. Curiously this subject has been very controversial historically [2].

\section{METHOD}

For comparison purposes coming up the magnitude of the upward centrifugal force, c.f., is estimated for the near surface region of the earth.

$$
c . f .=\frac{\rho v^{2}}{r}
$$

where $v$ is the speed tangent to the earth's surface, $r$ is the earth's radius and $\rho$ is the density of the surface material. Take the equatorial radius for $r$, the circumference in 24 hours for $v$, then the estimate for (1) is

$$
c . f .=\rho 3.38 \times 10^{-2} \mathrm{~m} / \mathrm{sec}^{2}
$$

which is a fraction of the force of gravity.

In the midst of rebounding from a continental boundary the tide causes a horizontal force back on the continent equal to the time rate of change of the linear momentum.

$$
F_{c}=\frac{2 \rho u}{T}
$$

where $u$ times the density is the linear momentum and $T$ is the time interval over which the momentum reversal takes place.

For a surface gravity wave propagating in an arbitrary mean depth $H$ the Stokes drift $u$ for depth $z$ is worked out in the hydrodynamics texts [3] is

$$
u=a^{2} \omega k \frac{\cosh 2 k(z+H)}{2 \sinh ^{2} k H}
$$

where $a$ and $k$ are the wave amplitude and wave number and $\omega$ is the wave frequency. Using known relations among hyperbolic sines and cosines and applying to the tide wave, and an approximation can be made reducing (4) to

$$
u=\frac{a^{2} \omega k}{2(k H)^{2}}
$$

where the approximation is that the tide wavelength is much larger than the mean water depth. In other words $k z$ and $K H$ are small quantities.

As a consequence (3) reduces to

$$
F_{c}=\frac{\rho}{T}\left(\frac{a}{H}\right)^{2} \frac{\omega}{k}
$$


Now consider $T$ is one half a wave period, $T=\pi / \omega$ and $c=\frac{\omega}{k}=\sqrt{g H}$ is the shallow water wave phase speed. Then (6) is

$$
F_{c}=\rho g\left(\frac{a}{H}\right)^{2}
$$

Due to the last two factors on the RHS of (7), each of which is small compared to one, that force is smaller than the force of gravity.

\section{DISCUSSION}

Depending on the variables selected the force on the continent could even be smaller in magnitude than the force in (2). That is not a problem. These two forces are not competing with each other. One is horizontal and the other vertical. A tendency of the centrifugal force is to decompress the upper surface layers of the earth. Otherwise why would the equatorial radius be in an expanded state? The force on the continent tends to compress what it comes in contact with which might even be a bit easier after the centrifugal force has done its work.

There is a concern about the reliability of the Stokes drift for arbitrary constant mean depth (4) brought in from the text books. It has been manufactured starting from the assumption of irrotational motion. That assumption has led in the past to incorrect results regarding surface gravity waves [4].

It is true that the continental boundaries are not vertical walls because of the shelves and slopes that border them. However, the tide wavelength is so great that it is reasonable to assume that reflection takes place as if there were vertical walls.

Tide reflection forces are maximum at full and new moon because that is when their amplitudes are greatest. That does not mean that an earthquake has to happen at such times. Many full moons come and go without any quakes occurring.

\section{CONCLUSION}

In October of 1989, a large earthquake struck Santa Cruz, CA three days after a full moon. During the intervening 30 years several times in several different places, it was noticed that a major earthquake occurred within one to three days of a full moon. Here, a mechanical model is advanced to help explain these observations: a compressive force caused by the tides reflecting off continental boundaries. It is calculated by taking the time rate of change of the linear momentum brought to the continent by the tide. For the linear momentum, the Stokes drift for a surface gravity wave propagating in an arbitrary mean depth, taken from the text books, is adapted to the tide wave. This horizontal compressive force can have the same magnitude as the vertical decompressive force that has been used to understand the expanded equatorial radius of the earth: the centrifugal force due to the earth's daily rotation about its axis.

\section{CONFLICTS OF INTEREST}

The authors declare no conflicts of interest regarding the publication of this paper.

\section{REFERENCES}

1. Barnett, T.P. and Kenyon, K.E. (1975) Recent Advances in the Study of Wind Waves. Reports on Progress in Physics, 38, 667-729. https://doi.org/10.1088/0034-4885/38/6/001

2. Kenyon, K.E. (2004) Force and Torque on a Wall from Reflected Surface Gravity Waves. Physics Essays, 17, 95-102. https://doi.org/10.4006/1.3025646

3. Kundu, P.K. (1990) Fluid Mechanics. Academic Press, San Diego, p. 225. 
4. Kenyon, K.E. (2021) Particle Motion in Surface Gravity Waves. Natural Science, 13, 18-20. https://doi.org/10.4236/ns.2021.132003 